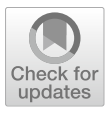

Cite as

Nano-Micro Lett.

(2021) 13:124

Received: 22 February 2021

Accepted: 14 April 2021

Published online: 11 May 2021

(C) The Author(s) 2021

\section{Highly Stretchable Shape Memory Self-Soldering Conductive Tape with Reversible Adhesion Switched by Temperature}

\title{
HIGHLIGHTS
}

- Shape memory self-soldering tape used as conductive interconnecting material.

- Perfect shape and conductivity memory performance and anti-fatigue performance.

- Reversible strong-to-weak adhesion switched by temperature.

\begin{abstract}
With practical interest in the future applications of next-generation electronic devices, it is imperative to develop new conductive interconnecting materials appropriate for modern electronic devices to replace traditional rigid solder tin and silver paste of high melting temperature or corrosive solvent requirements. Herein, we design highly stretchable shape memory self-soldering conductive (SMSC) tape with reversible adhesion switched by temperature, which is composed of silver particles encapsulated by shape memory polymer. SMSC tape has perfect shape and conductivity memory property and anti-fatigue ability even under the strain of $90 \%$. It also exhibits an initial conductivity of $2772 \mathrm{~S} \mathrm{~cm}^{-1}$ and a maximum tensile strain of $\sim 100 \%$. The maximum conductivity could be increased to $5446 \mathrm{~S} \mathrm{~cm}^{-1}$ by decreasing the strain to $17 \%$. Meanwhile, SMSC tape

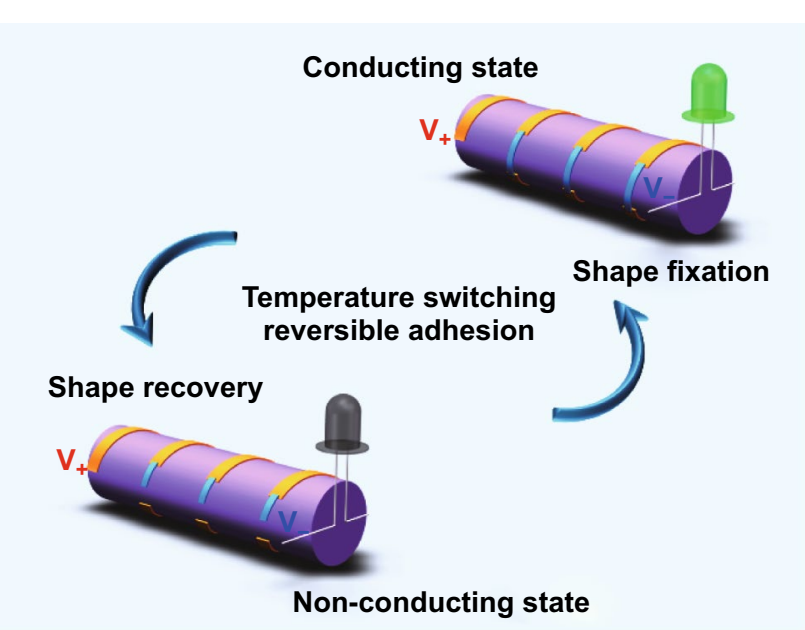
can easily realize a heating induced reversible strong-to-weak adhesion transition for self-soldering circuit. The combination of stable conductivity, excellent shape memory performance, and temperatureswitching reversible adhesion enables SMSC tape to serve two functions of electrode and solder simultaneously. This provides a new way for conductive interconnecting materials to meet requirements of modern electronic devices in the future.
\end{abstract}

KEYWORDS Shape memory performance; Self-soldering conductive tape; Reversible adhesion; Stretchable electronics

\footnotetext{
Mengyan Wang and Quan Zhang contributed equally to this work

$\triangle$ Rujun Ma,malab@nankai.edu.cn

1 School of Materials Science and Engineering, National Institute for Advanced Materials, Tianjin Key Lab for Rare Earth Materials and Applications, Nankai University, Tongyan Road 38, Tianjin 300350, People's Republic of China

2 State Key Laboratory and Institute of Elemento-Organic Chemistry, Centre of Nanoscale Science and Technology and Key Laboratory of Functional Polymer Materials, College of Chemistry, Nankai University, Weijin Road 94, Tianjin 300071, People’s Republic of China
} 


\section{Introduction}

In recent decades, modern electronic devices are developing toward integration, miniaturization, flexibility, scalability and biocompatibility [1-4]. Extensive studies on modern electronic devices focus on the functional elements, such as transistors [5, 6], sensors [7-11], electrodes [12-14], capacitors and batteries $[15,16]$, however, less attention has been paid to the related electronic interconnecting materials [17]. Among conventional electronic interconnecting materials, tin-based alloy solder and conductive adhesive are commonly used in modern electronic devices. In order to form a permanent conductive bond between the two elements, tin-based solder is first melted at a temperature higher than its melting temperature and then re-solidified at the connecting interface after cooling. High processing temperature may damage the substrate and temperature-sensitive elements. In contrast, epoxy-based commercial silver paste can form a good conductive way between various electronic elements after solvent volatilization, where it is not necessary for high temperature treatment [18]. Even so, the solvent in the paste may corrode the substrate and electronic elements. Meanwhile, the packaging structure with reduced volume for microelectronics has to face the reliability issue from the microscopic damage accumulation [19, 20]. Moreover, conventional electronic interconnecting materials are generally un-stretchable and may either crack at a strain as low as $1 \%$ or deform plastically at a strain greater than a few percent. These rigid and un-stretchable electronic interconnecting materials cannot meet the flexible requirements of wearable electronics. Therefore, it is imperative to develop new conductive interconnecting materials which possess stable conductivity, high stretchability, lower using condition and strong adhesion appropriate for modern electronic devices [18].

The most direct method to develop conductive interconnecting materials is to improve the existing interconnecting materials according to the demands of modern electronic devices. For example, tin-bismuth alloy solder with lower melting temperature can be used at processing temperature below 200 ${ }^{\circ} \mathrm{C}$ [21-23]. However, the defects such as segregation behavior and low adhesive strength hinder the application of tinbismuth alloy solder [24, 25]. In order to prevent the matrix and electronic components from being corroded by solvents, adhesives with different solvents were fabricated toward the specific needs of conductive materials [26-28]. Among them, the newly developed waterborne adhesive has a wider range of applications, while the oxidation and short circuit problems caused by water vapor need more attention [29-31]. In addition to the above two types of conductive interconnecting materials, researchers have also tried to develop various dry adhesives [32, 33]. Carbon nanotubes (CNTs) with hierarchical structures can provide an outstanding and stable adhesion for thermal and/or electric management [34, 35]. However, the relative research still stays in the laboratory due to the difficulties in the development of CNTs with ideal hierarchical structures. Another kind of dry adhesive realizes reversible adhesion based on shape memory effect [36-38]. The poor conductivity of shape memory polymer (SMP) is the biggest challenge to apply this dry adhesive for circuit repair. However, the characteristics of existing conductive interconnecting materials, such as high processing temperature, rigidness and corrosivity, are not compatible with the development of miniaturization, integration and flexibility of modern electronic devices. This mismatch limits the development of modern electronic devices. In order to fully play the role of modern electronic devices, it is particularly important to develop new conductive interconnecting materials.

In this work, we reported highly stretchable shape memory self-soldering conductive tape (SMSC tape) with reversible adhesion switched by temperature. The SMSC tape exhibited an initial conductivity of $2772 \mathrm{~S} \mathrm{~cm}^{-1}$ and a maximum tensile strain of $\sim 100 \%$. The maximum conductivity could be increased to $5446 \mathrm{~S} \mathrm{~cm}^{-1}$ by decreasing the strain to $17 \%$. It also had a perfect shape memory performance with strain fixation ratio and recovery ratio of $100 \%$. Meanwhile, there was no obvious degradation of shape memory performance after repeated stretching-recovery cycles. Thanks to the temperature induced shape recovery property, SMSC tape easily enabled reversible strong to weak adhesion transition. The combination of stable conductivity, excellent shape memory performance and reversible adhesion gives SMSC tape versatility to independently finish the collaborative work of multiple materials or cannot be completed by existing interconnecting materials.

\section{Experimental}

\subsection{Materials}

Silver acetate (99\%) was obtained from Adamas-beta, saturated ammonia solution (AR 25-28\%), formic acid aqueous solution (AR 88\%) and stearyl acrylate (SA) (> 96.0\% GC) 
were purchased from Aladdin. Urethane diacrylate (UDA) (CN9021 NS) was acquired from Sartomer. Benzoyl peroxide (BPO) (98\%) was purchased from MERYER. They were not purified before use.

\subsection{Synthesis of Silver Nanoparticles (Ag NPs)}

Ag NPs were prepared by thermal reduced method following a previous report [39]. Silver acetate $(1 \mathrm{~g})$ was dissolved in saturated ammonia solution $(10 \mathrm{~mL})$ by stirring at room temperature. After that, formic acid aqueous solution $(1 \mathrm{~mL})$ was added dropwise with continuous stirring. During this period, the solution turned muddy and then transparent. The transparent solution stood $12 \mathrm{~h}$ to settle down large Ag NPs. Then, the supernatant was transferred to a dry culture dish and heated at $70{ }^{\circ} \mathrm{C}$ for $1 \mathrm{~h}$. Further, the product was fully dried at $50{ }^{\circ} \mathrm{C}$ in the air. The dried product was washed by centrifugation using deionized water to remove impurities. Finally, the precipitation was fully dried at $60{ }^{\circ} \mathrm{C}$ in the air.

\subsection{Preparation of SMSC Tape}

SMSC tape was prepared by in situ formation of SA-UDA copolymer in blended composite under the condition of anaerobic heating. SA, UDA, BPO, and thermal reduced Ag NPs were proportionally mixed by vortex mixing using as the precursor of SMSC tape. After that, the precursor was degassed in a vacuum oven at $50{ }^{\circ} \mathrm{C}$. Then, the precursor was injected between a pair of warm glass sheets spaced with Kapton tape of $280 \mu \mathrm{m}$. The precursor was subsequently cured at $80{ }^{\circ} \mathrm{C}$ for $80 \mathrm{~min}$. Finally, the film was cooled to room temperature and gently peeled off from the glass sheets. The film can be further cut into required shape.

\subsection{Characterization and Evaluation}

The morphology of Ag NPs and SMSC tape was observed by SEM (JSM-7800F, JEOL). The structural information of Ag NPs and SMSC tape was characterized by XRD (Rigaku Smart Lab SE). DSC analysis was performed by the thermogravimetric analyzer (Netzsch STA 449F5) with a heating rate of $0.5^{\circ} \mathrm{C} \mathrm{min}^{-1}$. The tensile strain of SMSC tape was achieved by a home-made stress-strain test system, which was connected with a Keithley 2182A/6221 system to record all the electric signals in real-time. Two quantities, namely strain fixation ratio $\left(R_{f}\right)$ and strain recovery ratio $\left(R_{r}\right)$, were used to evaluated the shape memory performance of SMSC tape. They were, respectively, calculated as following:

$R_{f}=\frac{L_{f}-L_{0}}{L_{s}-L_{0}} \times 100 \%$

$R_{r}=\frac{L_{s}-L_{r}}{L_{s}-L_{0}} \times 100 \%$

where $L_{0}, L_{s}, L_{f}$, and $L_{r}$ are original length, tensile length, temporarily fixing length and final recovery length of SMSC tape, respectively. The shear adhesive strength of SMSC tape was also measured using home-made stress-strain measuring system. SMSC tape $\left(5 \times 5 \mathrm{~mm}^{2}\right)$ is sandwiched between two copper $(\mathrm{Cu})$ plates by pressing (preloading force of $\sim 4 \mathrm{kPa}$ ) at the heating state. Two $\mathrm{Cu}$ plates are fixed by the clamps in the measuring system, where the adhesive interface is parallel to the tensile direction. The load is recorded in realtime with the increase in distance between two clamps. The recorded maximum load before separation of two $\mathrm{Cu}$ plates is the corresponding shear strength. The output voltage and current of TENG made of SMSC tape were measured with a digital oscilloscope (DS1104, RIGOL) and a low noise electrometer (6514 system electrometer, Keithley).

\section{Results and Discussion}

\subsection{Tape Preparation and Characterization}

SMSC tape is prepared by anaerobic curing of well blended precursors, including SA, UDA, BPO, and Ag NPs (Figs. S1-S3). Under the condition of anaerobic heating, BPO induces radical reaction between SA and UDA to form the SMP in situ in composite. Meanwhile, the uniformly distributed Ag NPs construct a conductive network in the polymer. Annealing treatment further improves the electric conductivity of SMSC tape (Fig. 1a). At room temperature, SMSC tape can be cut into the required size. When it is heated above the melting temperature $\left(T_{m}\right)$, it is easily deformed to the desired shape. The temporary shape is maintained at room temperature until it is reheated to the melting temperature (Fig. 1b). Figure 1c is a digital optical image of an as-prepared SMSC tape on the glass substrate. The crosssectional scanning electron microscope (SEM) image shows that Ag NPs are uniformly dispersed in the cross-linked SAUDA SMP (Fig. 1d). The diameter center of the synthesized 
Ag NPs is $\sim 300 \mathrm{~nm}$. The X-ray diffraction (XRD) pattern is almost the same as the standard powder diffraction file (PDF) of 89-3722 Ag, which indicates that the synthesized Ag NPs have high crystalline. After annealing treatment, the crystallinity of Ag NPs do not change significantly (Fig. 1e). Compared with pure SA-UDA SMP, the melting temperature of SMSC tape is slightly higher and the melting enthalpy $\left(\Delta H_{m}\right)$ is greatly reduced. The former shows that Ag NPs do not decrease the crystallinity of SA-UDA copolymer, while the latter is due to the low specific heat of Ag NPs (Fig. 1f and Table S1).

\subsection{Electrical and Mechanical Properties}

Four comparative experiments in Fig. 2 analyzed the initial conductivity and maximum strain at $60{ }^{\circ} \mathrm{C}$ of SMSC tape synthesized by different formulations in detail. The weight ratio between SA and UDA is a key parameter that affects the conductivity and maximum strain of SMSC tape. As shown in Fig. 2a, the initial conductivity decreases with the mass ratio between SA and UDA from 40:60 (SA40UDA60) to 90:10 (SA90UDA10). Meanwhile, the maximum strain increases first and then decreases, where the inflection point is $80: 20$ (SA80UDA20). SA is less dense than UDA. This means that the same weight of SA is added into the SMSC tape instead of UDA to keep the total mass fraction of polymer constant in value, but the actual volume fraction of the polymer gradually increases as the SA content increases. The increase in polymer volume, namely the decrease of Ag NPs content, results in the decrease of SMSC tape conductivity. In addition, the maximum tensile strain of pure SMP made of SA and UDA decreases with the increase in SA content [40]. For the SMSC tape composed of polymer with 90:10 mass ratio of SA and UDA, the maximum strain decreases due to the decrease in intrinsic maximum strain of pure SMP, although the actual volume fraction of polymer (90:10 mass ratio) in SMSC tape is bigger than that with 80:20 mass ratio. To meet the requirements of conductivity and stretchability simultaneously, the mass ratio between SA and UDA is kept at a fixed value of 70:30 (SA70UDA30) to optimize other components and treatment conditions in SMSC tape. (a)

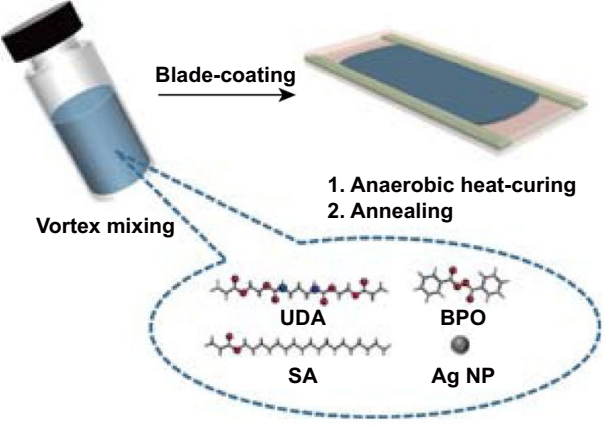

(c)

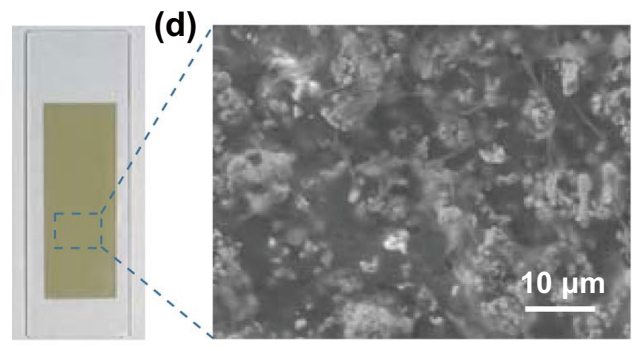

(b)

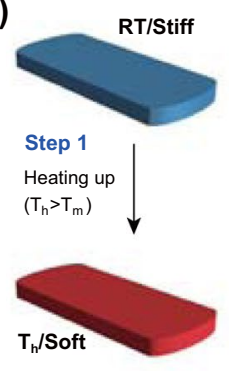

(e)

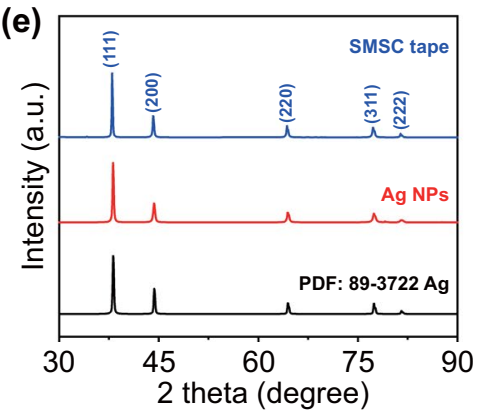

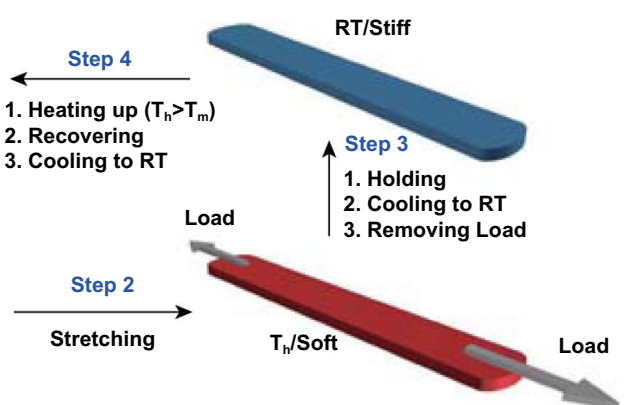

(f)

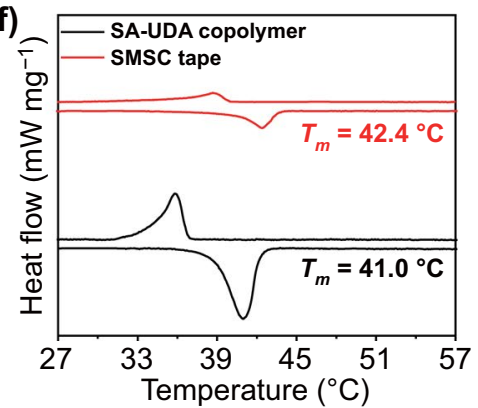

Fig. 1 Preparation and Characterization of SMSC tape. a Schematic illustration of the preparation process. b The shape memory effect of SMSC tape. c Optical image of as-prepared SMSC tape. d Cross-sectional morphology of SMSC tape. The scale bar is $10 \mu \mathrm{m}$. e XRD patterns of Ag NPs, SMSC tape and the powder diffraction file (PDF) of 89-3722 Ag. f DSC diagrams of SMSC tape (red line) and pure SA-UDA copolymer (black line) 

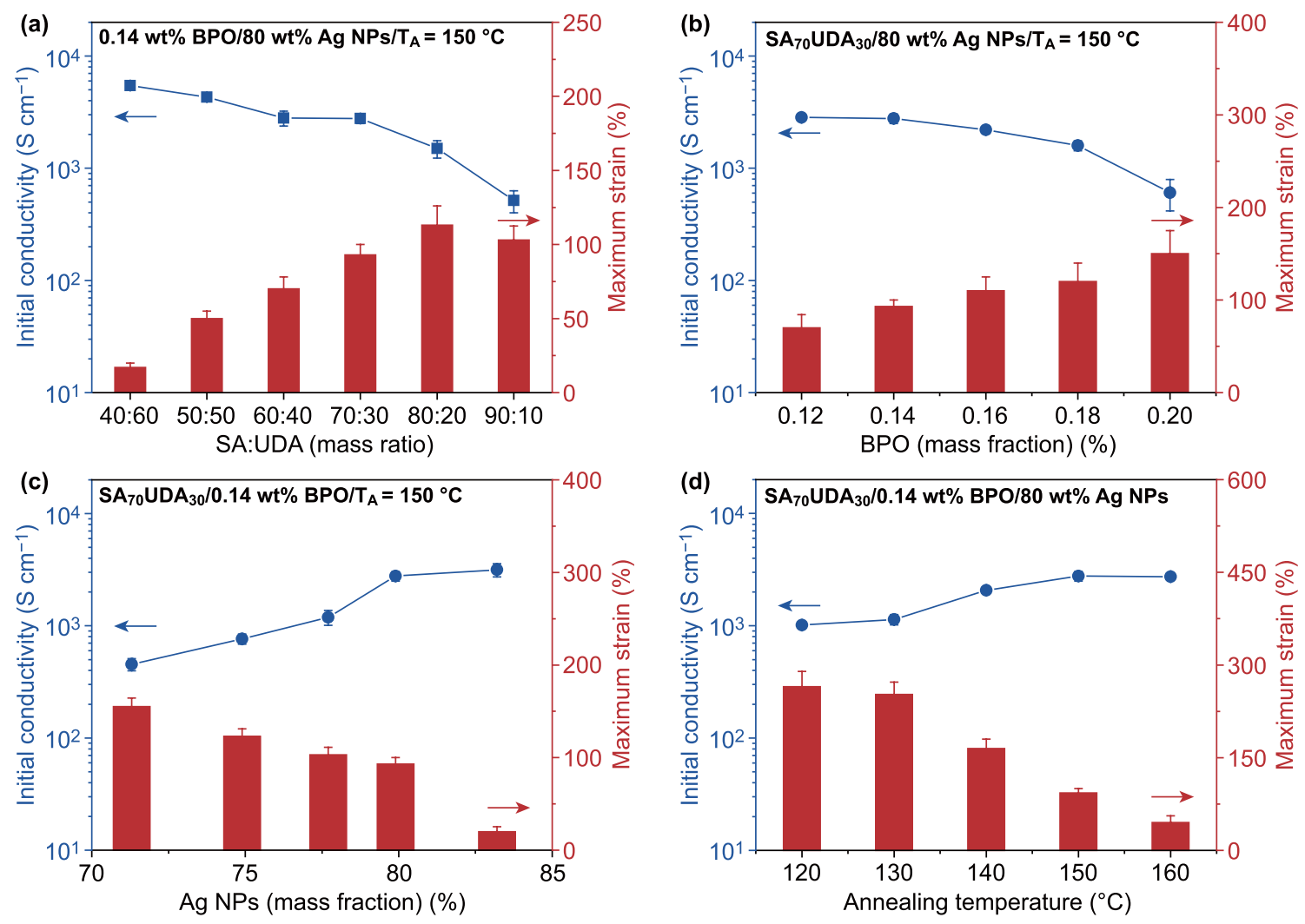

Fig. 2 Effects of four parameters on initial conductivity and maximum strain of SMSC tape at $60{ }^{\circ} \mathrm{C}$. $\mathbf{a}$ The mass ratio between SA and UDA. b The content of BPO. $\mathbf{c}$ The content of Ag NPs. $\mathbf{d}$ Annealing temperature

Another important factor to consider is the content of BPO. Here, BPO acts as radical initiator to promote radical reaction of SA and UDA under the condition of anaerobic heating. Thus, more BPO can induce more dramatic polymerization between SA and UDA in the same curing period of time. In other words, more polymer chains with high molecular weight are formed in the SMSC tape. The existence of these long-chain molecules is conducive to the ductility of SMSC tape. On the other hand, they hinder the formation of conductive paths in SMSC tape. As shown in Fig. 2b, the initial conductivity of the SMSC tape decreases with the increase of BPO content, but the maximum strain gradually increases.

The content of Ag NPs directly determines the conductivity of the SMSC tape. The more Ag NPs are incorporated into the composite, the higher conductivity is reached. Figure $2 \mathrm{c}$ shows that the initial conductivity significantly increases with the increase in Ag NPs content, while the maximum strain decreases. Under the premise of sufficient Ag NPs content, the annealing treatment can improve the electric contact between adjacent Ag NPs. As the annealing temperature $\left(T_{A}\right)$ rises, the initial conductivity increases, but the maximum strain decreases (Fig. 2d). In general, there is a trade-off between the initial conductivity and the maximum tensile strain of the SMSC tape. In essence, it is a balance between conductive network that improves conductivity and interaction of molecule chain for increasing stretchability.

\subsection{Shape-Memory Performance}

SMSC tape containing SA-UDA copolymer encapsulated Ag NPs exhibits almost perfect shape memory performance. Here, $150{ }^{\circ} \mathrm{C}$ annealed SMSC tape containing $19.86 \mathrm{wt} \% \mathrm{SA}_{70} \mathrm{UDA}_{30}$ copolymer, $80 \mathrm{wt} \% \mathrm{Ag}$ NPs and $0.14 \mathrm{wt} \% \mathrm{BPO}$ is used to demonstrate the recovery capacity of conductivity and shape memory effect. It has an initial conductivity of $2772 \mathrm{~S} \mathrm{~cm}^{-1}$ and a maximum strain of $\sim 100 \%$. The conductivity gradually decreases with the tensile strain and it is still $1618 \mathrm{~S} \mathrm{~cm}^{-1}$ at $90 \%$ (Fig. 3a). At $60^{\circ} \mathrm{C}$, the elastic modulus of SMSC tape is 
$0.36 \mathrm{MPa}$ and the maximum tensile stress before fracture is only $0.20 \mathrm{MPa}$, both of which are much lower than those of shape memory alloy. In contrast, the elastic modulus and the maximum tensile stress of SMSC tape at room temperature are 52.2 and 3.7 MPa, respectively (Fig. S4 and Table S1) [41]. In the heated state, such a low elastic modulus and maximum tensile stress make the shape of the SMSC tape easy to change without a large external load. The strong mechanical strength at room temperature enables SMSC tape to resist the external impact. Strain fixation ratio $\left(R_{f}\right)$ and strain recovery ratio $\left(R_{r}\right)$ are two important quantities for evaluating shape-memory performance. They are calculated using equations described in experimental methods. The former has the ability of switching segments (SA) to fix the temporary shape, while the latter is used to describe the ability of the shape-memory segment (UDA) to memorize the permanent shape. Figure $3 \mathrm{~b}$ shows $R_{f}$ and $R_{r}$ of SMSC tape as a function of tensile strain. Under different strains from 10 to $90 \%$, the SMSC tape shows perfect ability of shape-fixation and shape-recovery, namely almost
$100 \% R_{f}$ and $100 \% R_{r}($ Video $\mathrm{S} 1)$. The insets in Fig. 3b give a visual representation of the perfect shape-fixation at $90 \%$ strain and shape-recovery.

SMSC tape shows good recovery capacity of conductivity thanks to its excellent shape memory performance. As shown in Fig. 3c, there is no obvious hysteresis in conductivity during a stretching and releasing process. With the repeating stretching-releasing cycles, the conductivity of SMSC tape decreases and gradually becomes a stable value, when maximum strain is $20 \%$ (Fig. S5). Furthermore, we evaluated the fatigue resistance capability in shape memory performance of SMSC tape (Fig. 3d). When the maximum strain is $50 \%, R_{f}$ has always maintained $100 \%$ after 200 cycles, which indicates that the SMSC tape has near perfect anti-fatigue performance in shape-fixation ability. Meanwhile, when the number of cycles is less than 100 times, $R_{r}$ is also $100 \%$. And from 100 to 200 th cycles, the degradation of $R_{r}$ decreases from 100 to $99.93 \%$. However, due to the recoverable conductivity and near perfect shape memory
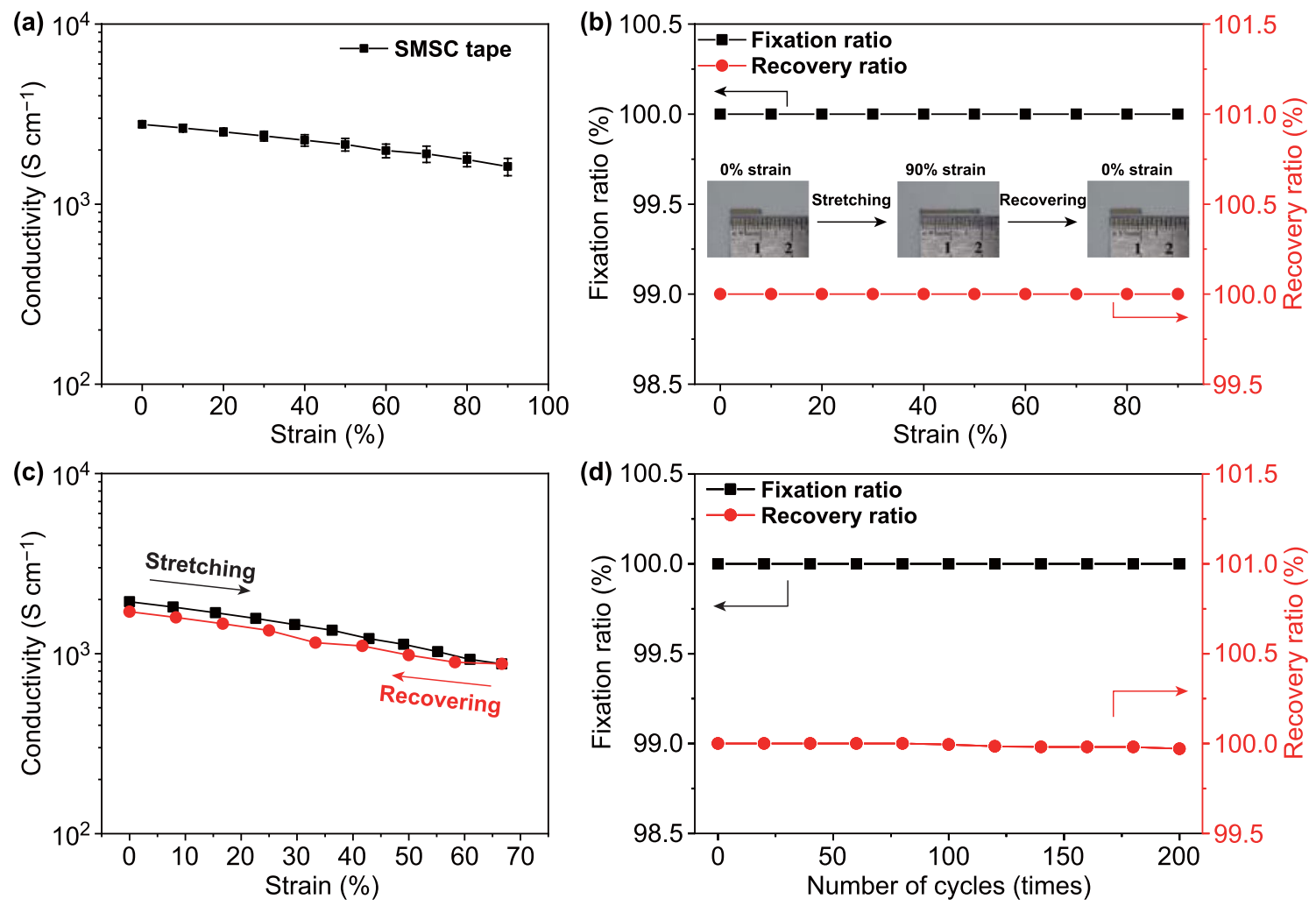

Fig. 3 Shape-memory performance of SMSC tape. a Conductivity of SMSC tape at room temperature as a function of tensile strain. $\mathbf{b} R_{f}$ and $R_{r}$ of shape fixing and recovery of SMSC tape at different tensile strains. c A cyclic conductivity-strain test is carried out using SMSC tape at maximum strain of $66.7 \%$. d $R_{f}$ and $R_{r}$ of cyclic shape fixing and recovery of SMSC tape at tensile strain of 50\%. The mass ratio between SA and UDA is $70: 30$. The mass fraction of Ag NPs and BPO is $80 \%$ and $0.14 \%$, respectively. The annealing temperature is $150{ }^{\circ} \mathrm{C}$ 
performance, SMSC tape can be easily reused many times in practice.

\subsection{Temperature-Dependent Reversible Adhesion}

Besides good conductivity and perfect shape memory performance, SMSC tape also shows temperature-dependent reversible adhesion. SMSC tape is rigid at room temperature, which is unable to obviously wet the surface. Thus, it has no adhesion ability. When the SMSC tape is heated above the melting temperature, the SMSC tape softens and gradually wets the surface under a moderate pressure, resulting in sharp increase in adhesion upon cooling (Fig. 4a). Figure $4 b$ shows the shear adhesive strength of SMSC tape in the cooling state as a function of heating temperature. When the temperature is equal to or lower than $40^{\circ} \mathrm{C}$, the shear adhesive strength is almost zero. Once the heating temperature is higher than $45^{\circ} \mathrm{C}$, which is larger than the melting temperature, the shear adhesive strength of SMSC tape increases significantly and reaches to the highest value $(\sim 0.43 \mathrm{MPa})$ after heating at $70^{\circ} \mathrm{C}$. As the heating temperature increases further, shear adhesive strength gradually decreases due to the excessive softening of SMSC tape (Fig. S6). We also measured the shear adhesive strength of SMSC tape (only 0.02 $\mathrm{MPa})$ at heating state $\left(70^{\circ} \mathrm{C}\right)$, which was significantly lower than that in cooling state. The shear adhesive strength evolution of SMSC tape during 200 cycles of repeated attaching-peeling with $70{ }^{\circ} \mathrm{C}$ heating temperature is shown in Fig. 4c. There is no obvious increase or decrease in shear adhesive strength under heating and cooling state. Strong shear adhesive strength in the cooling state can meet the requirement of connection between two terminals, while the weak shear adhesive strength in the heating state make it easy to peel off the SMSC tape. Meanwhile, due to reversible and stable adhesion, SMSC tape is not disposable but recyclable. SMSC tape can also provide extraordinary adhesion on various substrates, especially $0.42 \mathrm{MPa}$ on $\mathrm{Cu}$ plate, which is even stronger than that of commercial conductive tape (Fig. 4d). Different from commercial adhesives, the stickiness of SMSC tape is the embedded structure between SMSC tape and the substrate in essence, rather than the molecular interaction between the macromolecules in the adhesive and the target materials [37]. Thus, the surface roughness of the object is an important factor influencing the adhesive strength of SMSC tape on the object. Compared with ITO and aluminum (Al) foil, the surface of $\mathrm{Cu}$ plate is rougher. It leads to the adhesive strength

(a)
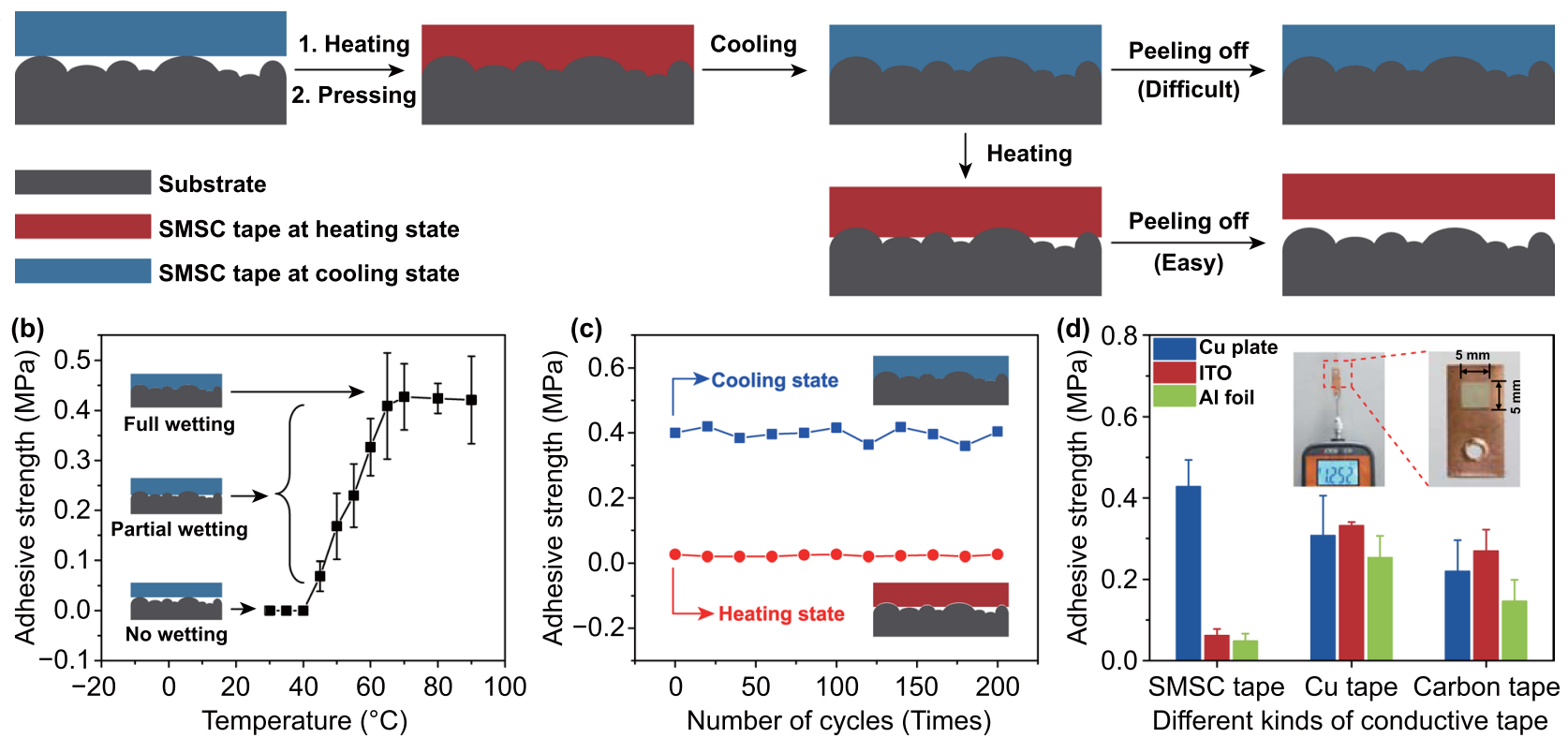

Fig. 4 Reversible adhesion of SMSC tape switched by temperature. a Schematic illustration of adhesion reversible process of SMSC tape switched by temperature. b Shear adhesive strength between SMSC tape and Cu plate at room temperature switched by different heating temperature. Inset is the wetting state of SMSC tape at cooling state on rough substrate with the different heating temperature. c Reversible shear adhesive strength of SMSC tape between heating state and cooling state as a function of the number of cycles. The switching temperature is 70 ${ }^{\circ} \mathrm{C}$. d Shear adhesive strength of SMSC tape and two kinds of commercial conductive tape on various conductive surfaces. The inset is a digital image illustrating the adhesion force of SMSC tape on the $\mathrm{Cu}$ plate 
of SMSC tape on $\mathrm{Cu}$ plate much stronger than those on ITO and $\mathrm{Al}$ foil. As shown in the inset of Fig. $4 \mathrm{~d}$, SMSC tape $\left(5 \times 5 \mathrm{~mm}^{2}\right)$ attached on $\mathrm{Cu}$ plate can withstand a load of $1.252 \mathrm{~kg}$ in the shear direction (corresponding to $0.50 \mathrm{MPa}$ ). The site of fracture is located at the interface between SMSC tape and $\mathrm{Cu}$ plate, not the SMSC tape. This may be due to the fact that the maximum tensile strength of SMSC tape is much larger than the adhesive strength between $\mathrm{Cu}$ plate and SMSC tape [42]. The adhesive strength of SMSC tape exhibits an outstanding fatigue resistance performance. As shown in Fig. S7, there is no obvious degradation in adhesive strength and electric contact between $\mathrm{Cu}$ plate and SMSC tape after 500 times of applying-releasing shear load. In addition, this adhesive strength is affected by environmental temperature. As the environmental temperature increases, the adhesive strength of the interface between $\mathrm{Cu}$ plate and SMSC tape gradually decreases, along with the decline in its conductivity (Fig. S8). Once the environmental temperature is greater than the melting temperature of SMSC tape, the $\mathrm{Cu}$ plate and SMSC tape will be completely separated under a small load. It is worth noting that this thermal fatigue is caused by softening of SMSC tape under high temperature, which is different from the coefficient of thermal expansion mismatch commonly used for interconnecting materials [43, 44].

The content of each component in SMSC tape and annealing temperature can directly affect the intrinsic adhesive strength. Shear adhesive strength of SMSC tape synthesized by different formulations has been compared in Fig. S9. When the mass ratio between SA and UDA changes from 40:60 to 90:10, the adhesive strength increases first and then decreases. Similarly, it also increases first and then decreases with the increase of $\mathrm{Ag}$ NPs content in the polymer. On the other hand, with the increase in BPO content and annealing temperature, the shear adhesive strength of SMSC tape decreases. However, both the optimization of component content and the change of annealing temperature essentially change the wetting behavior of SMSC tape in the heating state, thus determining the intrinsic adhesive strength of SMSC tape.

\subsection{Potential Applications}

Because of the advantages of stable conductivity, perfect shape memory property and temperature switching reversible adhesion, SMSC tape can independently accomplish the task that requires cooperation of multiple materials or cannot be finished by existing electronic interconnecting materials. In general, a conductor is fixed between two terminals by solder or silver paste to make electric current conduct, while SMSC tape can fix itself on the $\mathrm{Cu}$ plate by its own adhesion to conduct the circuit (Fig. 5a). The solution in silver paste and the high temperature required to melt solder present potentially hazards to circuits, especially complex and highly integrated printed circuit board, which challenges the soldering skills of professionals. By contrast, an inexperienced person can easily repair the circuit through SMSC tape. Just a 50-60 ${ }^{\circ} \mathrm{C}$ heating for several seconds (such as offered by a hair dryer) and finger pressing are all that it takes to relight up the light-emitting diode (LED) (Figs. 5b and S10). Meanwhile, SMSC tape is easy to remove from the $\mathrm{Cu}$ substrate after reheating and no residue leaves on the substrate (Video S2). Even if the distance between the two $\mathrm{Cu}$ plates is larger than the original length of SMSC tape, the circuit still can be easily repaired by stretching SMSC tape (Fig. 5c). SMSC tape is also shaped into a bending pattern on a cylindrical substrate. The bending deformation has no effect on the brightness of the LED (Fig. 5d).

SMSC tape is also able to handle the responsibility of electrode by itself. A SMSC tape-based triboelectric nanogenerator (TENG) working in two-electrode mode is prepared. SMSC tape and polytetrafluoroethylene (PTFE) film are used as negative and positive triboelectric layer, respectively. Moreover, SMSC tape is used as an electrode due to its conductivity. A $\mathrm{Cu}$ plate is attached on the back of PTFE film to collect the current from the PTFE film (Fig. 5e). SMSC tape-based TENG driven by manual tapping can light up at least 42 commercial LEDs (Video S3). The triboelectric performance of SMSC tape-based TENG is investigated, when SMSC tape is fixed at different tensile strains in a stretching-releasing cycle. The results show that the output voltage and current (under a stress of $\sim 0.2$ $\mathrm{MPa}$ at a frequency of $5 \mathrm{~Hz}$ ) are almost unchanged (Fig. 5f, g). These experimental results indicate that SMSC tape is a promising candidate as a novel conductive connecting material for applications in the next generation of electronic devices in the future. 
(a)

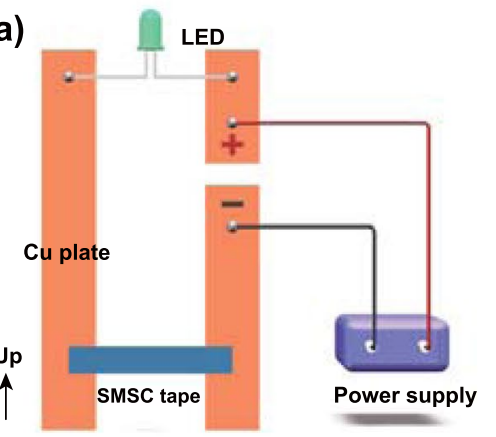

(e)

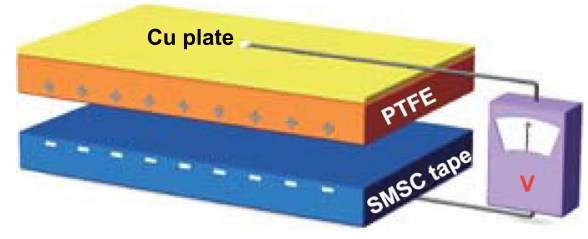

(b)
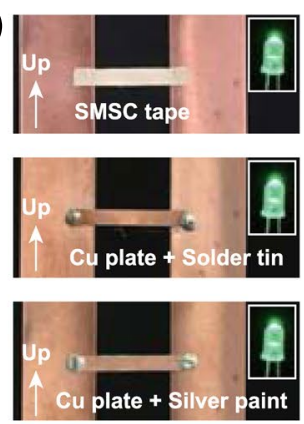

(f) 50

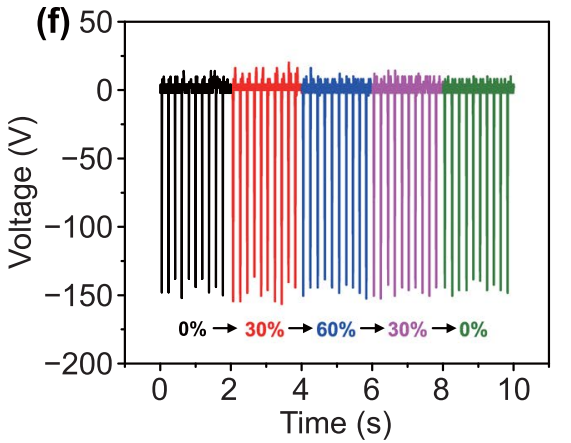

(c)
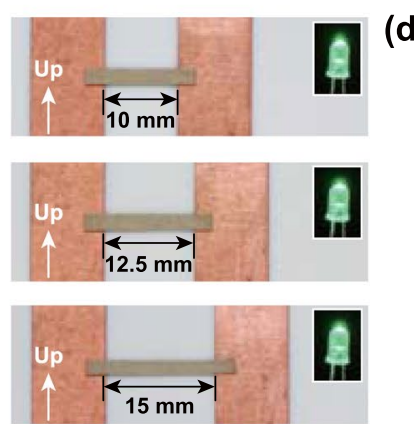

(d)

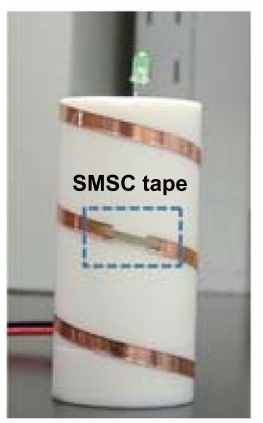

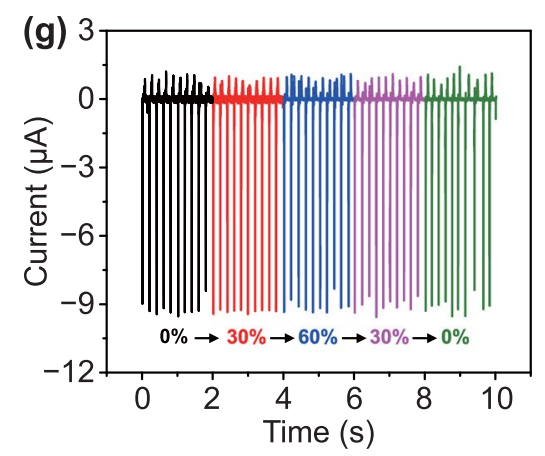

Fig. 5 Potential applications of SMSC tape. a Schematic illustration of an LED in a simple series circuit powered by a direct-current power supply. b Comparison in brightness of the working LEDs, where the circuits are repaired by SMSC tape, $\mathrm{Cu}$ plate and solder, and Cu plate and silver paste, respectively. $\mathbf{c}$ Comparison in brightness of the working LEDs, where the circuits are repaired by SMSC tape with different tensile strain. $\mathbf{d}$ Optical image of a circuit used to light LED. The circuit is repaired by bending SMSC tape. e Schematic illustration of a SMSC tape-based TENG. f Output voltage and $\mathbf{g}$ current of SMSC tape-based TENG when SMSC tape is fixed at different tensile strains in a stretching-releasing cycle

\section{Conclusion}

In summary, we designed a highly stretchable shape memory self-soldering conductive tape with reversible adhesion switched by temperature. The tape is prepared by anaerobic curing of well blended precursors, including SA, UDA, BPO and Ag NPs. A series of experimental results show that SMSC tape exhibits an initial conductivity of $\sim 2772 \mathrm{~S} \mathrm{~cm}^{-1}$ and a maximum strain of $\sim 100 \%$. With the increase in Ag NPs content, the initial conductivity could be increased to $5446 \mathrm{~S} \mathrm{~cm}^{-1}$ by decreasing the strain to $17 \%$. Meanwhile, SMSC tape has the perfect shape memory performance and fatigue resistance ability. The perfect shape memory effect gives SMSC tape a temperaturedetermined reversible strong-weak adhesion transition. The adhesive strength of SMSC tape can be compared with the commercial conductive tape, where the adhesive mechanism is from the embedded structure rather than the molecular interaction. Thanks to the stable conductivity, perfect shape memory performance and reversible self-adhesion, SMSC tape can serve two functions of electrode and solder simultaneously. As a new type of conductive interconnecting material, SMSC tape possesses the characteristics of no adhesive residue and low processing temperature, and has promising compatibility with modern electronic devices. It also provides a unique idea for the development of electronic devices in the future.

Acknowledgements This work is supported by National Key R\&D Program of China (Grant No. 2020YFA0711500), the National Natural Science Fund of China (51973095 \& 52011540401).

Open Access This article is licensed under a Creative Commons Attribution 4.0 International License, which permits use, sharing, adaptation, distribution and reproduction in any medium or format, as long as you give appropriate credit to the original author(s) and the source, provide a link to the Creative Commons licence, and indicate if changes were made. The images or other third party material in this article are included in the article's Creative Commons licence, unless indicated otherwise in a credit line to the material. If material is not included in the article's Creative Commons licence and your intended use is not permitted by statutory regulation or exceeds the permitted use, you will need to obtain permission directly from the copyright holder. To view a copy of this licence, visit http://creativecommons.org/licenses/by/4.0/. 
Supplementary Information The online version contains supplementary material available at https://doi.org/10.1007/ s40820-021-00652-0.

\section{References}

1. H. Joo, D. Jung, S.H. Sunwoo, J.H. Koo, D.H. Kim, Material design and fabrication strategies for stretchable metallic nanocomposites. Small 16, 1906270 (2020). https://doi.org/ 10.1002/smll.201906270

2. S. Choi, S.I. Han, D. Jung, H.J. Hwang, C. Lim et al., Highly conductive, stretchable and biocompatible Ag-Au core-sheath nanowire composite for wearable and implantable bioelectronics. Nat. Nanotechnol. 13, 1048-1056 (2018). https://doi.org/ 10.1038/s41565-018-0226-8

3. R. Ma, S. Chou, Y. Xie, Q. Pei, Morphological/nanostructural control toward intrinsically stretchable organic electronics. Chem. Soc. Rev. 48, 1741-1786 (2019). https://doi.org/10. 1039/c8cs00834e

4. R. Ma, B. Kang, S. Cho, M. Choi, S. Baik, Extraordinarily high conductivity of stretchable fibers of polyurethane and silver nanoflowers. ACS Nano 9, 10876-10886 (2015). https:// doi.org/10.1021/acsnano.5b03864

5. J. Liang, K. Tong, Q. Pei, A water-based silver-nanowire screen-print ink for the fabrication of stretchable conductors and wearable thin-film transistors. Adv. Mater. 28, 5986-5996 (2016). https://doi.org/10.1002/adma.201600772

6. N. Matsuhisa, M. Kaltenbrunner, T. Yokota, H. Jinno, K. Kuribara et al., Printable elastic conductors with a high conductivity for electronic textile applications. Nat. Commun. 6, 7461 (2015). https://doi.org/10.1038/ncomms8461

7. J. Lee, H. Kwon, J. Seo, S. Shin, J.H. Koo et al., Conductive fiber-based ultrasensitive textile pressure sensor for wearable electronics. Adv. Mater. 27, 2433-2439 (2015). https://doi.org/ 10.1002/adma.201500009

8. B. Tian, Q. Liu, C. Luo, Y. Feng, W. Wu, Multifunctional ultrastretchable printed soft electronic devices for wearable applications. Adv. Electron. Mater. 6, 1900922 (2020). https:// doi.org/10.1002/aelm.202070006

9. N. Matsuhisa, D. Inoue, P. Zalar, H. Jin, Y. Matsuba et al., Printable elastic conductors by in situ formation of silver nanoparticles from silver flakes. Nat. Mater. 16, 834-840 (2017). https://doi.org/10.1038/NMAT4904

10. C.S. Boland, U. Khan, G. Ryan, S. Barwich, R. Charifou et al., Sensitive electromechanical sensors using viscoelastic graphene-polymer nanocomposites. Science 354, 1257-1260 (2016). https://doi.org/10.1126/science.aag2879

11. M. Park, J. Im, M. Shin, Y. Min, J. Park et al., Highly stretchable electric circuits from a composite material of silver nanoparticles and elastomeric fibres. Nat. Nanotechnol. 7, 803-809 (2012). https://doi.org/10.1038/nnano.2012.206

12. X. Wu, Y. Han, X. Zhang, C. Lu, Spirally structured conductive composites for highly stretchable, robust conductors and sensors. ACS Appl. Mater. Interfaces 9, 23007-23016 (2017). https://doi.org/10.1021/acsami.7b06256
13. H.S. Lee, Y. Jo, J.H. Joo, K. Woo, Z. Zhong et al., Threedimensionally printed stretchable conductors from surfactantmediated composite pastes. ACS Appl. Mater. Interfaces 11, 12622-12631 (2019). https://doi.org/10.1021/acsami.8b21570

14. Y. Kim, J. Zhu, B. Yeom, M. Di Prima, X. Su et al., Stretchable nanoparticle conductors with self-organized conductive pathways. Nature 500, 59-63 (2013). https://doi.org/10.1038/ nature 12401

15. H. Li, J. Liang, Recent development of printed micro-supercapacitors: printable materials, printing technologies, and perspectives. Adv. Mater. 32, 1805864 (2019). https://doi.org/10. 1002/adma.201805864

16. M.R. Lukatskaya, B. Dunn, Y. Gogotsi, Multidimensional materials and device architectures for future hybrid energy storage. Nat. Commun. 7, 12647 (2016). https://doi.org/10. 1038/ncomms12647

17. Z. Yang, Z. Zhai, Z. Song, Y. Wu, J. Liang et al., Conductive and elastic $3 \mathrm{~d}$ helical fibers for use in washable and wearable electronics. Adv. Mater. 32, 1907495 (2020). https://doi.org/ 10.1002/adma.201907495

18. R. Aradhana, S. Mohanty, S.K. Nayak, A review on epoxybased electrically conductive adhesives. Int J. Adhes. Adhes. 99, 102596 (2020). https://doi.org/10.1016/j.ijadhadh.2020. 102596

19. X. Long, Q. Jia, Z. Li, S.X. Wen, Reverse analysis of constitutive properties of sintered silver particles from nanoindentations. Int. J. Solids Struct. 191, 351-362 (2020). https://doi. org/10.1016/j.ijsolstr.2020.01.014

20. X. Long, B. Hu, Y. Feng, C. Chang, M. Li, Correlation of microstructure and constitutive behaviour of sintered silver particles via nanoindentation. Int. J. Mech. Sci. 161, 105020 (2019). https://doi.org/10.1016/j.ijmecsci.2019.105020

21. T. Heckmann, T. Souvignet, S. Lepeer, D. Naccache, Lowtemperature low-cost 58 Bismuth-42 Tin alloy forensic chip re-balling and re-soldering. Digit. Investig. 19, 60-68 (2016). https://doi.org/10.1016/j.diin.2016.10.003

22. L.E. Felton, C.H. Raeder, D.B. Knorr, The properties of tinbismuth alloy solders. JOM 45, 28-32 (1993). https://doi.org/ 10.1007/BF03222377

23. N.A. Ezaham, N.R.A. Razak, M.A.A.M. Salleh, Influence of bismuth on the solidification of tin copper lead-free solder alloy. AIP Conf. Proc. 2045, 020104 (2018). https://doi.org/ 10.1063/1.5080917

24. T. Satoh, T. Ishizaki, M. Usui, Effect of bismuth-tin alloy particle diameter on bonding strength of copper nanoparticles/bismuth-tin solder hybrid joints. J. Mater. Sci. Mater. Electron. 29, 7161-7176 (2018). https://doi.org/10.1007/ s10854-018-8704-1

25. S. Jin, M. Kim, S. Kanayama, H. Nishikawa, Microstructure and mechanical properties of indium-bismuth alloys for low melting-temperature solder. J. Mater. Sci. Mater. Electron. 29, 16460-16468 (2018). https://doi.org/10.1007/ s10854-018-9738-0

26. R. Ma, S. Kwon, Q. Zheng, H.Y. Kwon, J.I. Kim, Carbonnanotube/silver networks in nitrile butadiene rubber for highly conductive flexible adhesives. Adv. Mater. 24, 3344-3349 (2012). https://doi.org/10.1002/adma.201201273 
27. G. Cao, L. Wang, Y. Tian, Highly dispersed polypyrrole nanotubes for improving the conductivity of electrically conductive adhesives. J. Mater. Sci. Mater. Electron. 31, 9675-9684 (2020). https://doi.org/10.1007/s10854-020-03513-5

28. Q. Yan, M. Zhou, H. Fu, A reversible and highly conductive adhesive: towards self-healing and recyclable flexible electronics. J. Mater. Chem. C 8, 7772-7785 (2020). https://doi.org/ 10.1039/C9TC06765E

29. F. Wang, L. Feng, Y. Huang, W. Shen, H. Ma, Effect of the gradient distribution of multiwalled carbon nanotubes on the bond strength and corrosion resistance of waterborne polyurethane conductive nanocomposites. Prog. Org. Coat. 140, 105507 (2020). https://doi.org/10.1016/j.porgcoat.2019. 105507

30. B. Kim, T. Park, J. Kim, E. Kim, Waterborne polyacrylic/ PEDOT nanocomposites for conductive transparent adhesives. J. Nanosci. Nanotechnol. 13, 7631-7636 (2013). https://doi. org/10.1166/jnn.2013.7822

31. T. Wang, C.H. Lei, A.B. Dalton, C. Creton, Y. Lin et al., Waterborne, nanocomposite pressure-sensitive adhesives with high tack energy, optical transparency, and electrical conductivity. Adv. Mater. 18, 2730-2734 (2006). https://doi.org/10. 1002/adma.200601335

32. J.M. Krahn, A.G. Pattantyus-Abraham, C. Menon, Polymeric electro-dry-adhesives for use on conducting surfaces. Proc. Inst. Mech. Eng. Pt. L-J. Mater. Design Appl. 228, 109-114 (2014). https://doi.org/10.1177/1464420713509376

33. Y. Ko, J. Oh, K.T. Park, S. Kim, W. Huh et al., Stretchable conductive adhesives with superior electrical stability as printable interconnects in washable textile electronics. ACS Appl. Mater. Interfaces 11, 37043-37050 (2019). https://doi.org/10. 1021/acsami.9b11557

34. L. Qu, L. Dai, M. Stone, Z. Xia, Z.L. Wang, Carbon nanotube arrays with strong shear binding-on and easy normal liftingoff. Science 322, 238-242 (2008). https://doi.org/10.1126/ science. 1159503

35. M. Xu, F. Du, S. Ganguli, A. Roy, L. Dai, Carbon nanotube dry adhesives with temperature-enhanced adhesion over a large temperature range. Nat. Commun. 7, 13450 (2016). https://doi.org/10.1038/ncomms 13450
36. Y. Wang, H. Lai, Z. Cheng, H. Zhang, Y. Liu et al., Smart superhydrophobic shape memory adhesive surface toward selective capture/release of microdroplets. ACS Appl. Mater. Interfaces 11, 10988-10997 (2019). https://doi.org/10.1021/ acsami.9b00278

37. J. Dong, W. Zou, F. Chen, Q. Zhao, A soft shape memory reversible dry adhesive. Chin. J. Polym. Sci. 36, 953-959 (2018). https://doi.org/10.1007/s10118-018-2119-6

38. H. Zhang, H. Lai, Z. Cheng, D. Zhang, P. Liu et al., In-situ switchable superhydrophobic shape memory microstructure patterns with reversible wettability and adhesion. Appl. Surf. Sci. 525, 146525 (2020). https://doi.org/10.1016/j.apsusc. 2020.146525

39. C.M. Ajmal, S. Bae, S. Baik, A superior method for constructing electrical percolation network of nanocomposite fibers: in situ thermally reduced silver nanoparticles. Small 15, 1803255 (2019). https://doi.org/10.1002/smll.201803255

40. Z. Ren, W. Hu, C. Liu, S. Li, X. Niu et al., Phase-changing bistable electroactive polymer exhibiting sharp rigid-to-rubbery transition. Macromolecules 49, 134-140 (2016). https:// doi.org/10.1021/acs.macromol.5b02382

41. R. Matsui, K. Takeda, H. Tobushi, E. Pieczyska, Mechanical properties and advanced subjects in shape memory alloys and polymers. J. Theor. Appl. Mech. 56, 447-456 (2018). https:// doi.org/10.15632/jtam-pl.56.2.44

42. X. Long, Y. Wang, L.M. Keer, Y. Yao, Mechanical effects of isolated defects within a lead-free solder bump subjected to coupled thermal-electrical loading. J. Micromechanics Mol. Phys. 01, 1650004 (2016). https://doi.org/10.1142/S2424 913016500041

43. W. Wang, Z. Chen, S. Wang, X. Long, Mechanics-based acceleration for estimating thermal fatigue life of electronic packaging structure. Microelectron. Reliab. 107, 113616 (2020). https://doi.org/10.1016/j.microrel.2020.113616

44. X. Long, Y. Liu, F. Jia, Y. Wu, Y. Fu et al., Thermal fatigue life of $\mathrm{Sn}-3.0 \mathrm{Ag}-0.5 \mathrm{Cu}$ solder joint under temperature cycling coupled with electric current. J. Mater. Sci. Mater. El. 30, 7654-7664 (2019). https://doi.org/10.1007/ s10854-019-01081-x 